\title{
Simulated Nanoscale Peeling Process of Monolayer Graphene Sheet: Effect of Edge Structure and Lifting Position
}

\author{
Naruo Sasaki, ${ }^{1}$ Hideaki Okamoto, ${ }^{1}$ Shingen Masuda, ${ }^{1}$ Kouji Miura, ${ }^{2}$ and Noriaki Itamura ${ }^{1}$ \\ ${ }^{1}$ Department of Materials and Life Science, Faculty of Science and Technology, Seikei University, 3-3-1 Kichijoji-Kitamachi, \\ Musashino-shi, Tokyo 180-8633, Japan \\ ${ }^{2}$ Department of Physics, Aichi University of Education, Hirosawa 1, Igayacho, Kariya-shi, Aichi 448-8542, Japan
}

Correspondence should be addressed to Naruo Sasaki, naru@st.seikei.ac.jp

Received 1 June 2010; Accepted 4 August 2010

Academic Editor: Rakesh Joshi

Copyright (C) 2010 Naruo Sasaki et al. This is an open access article distributed under the Creative Commons Attribution License, which permits unrestricted use, distribution, and reproduction in any medium, provided the original work is properly cited.

\begin{abstract}
The nanoscale peeling of the graphene sheet on the graphite surface is numerically studied by molecular mechanics simulation. For center-lifting case, the successive partial peelings of the graphene around the lifting center appear as discrete jumps in the force curve, which induce the arched deformation of the graphene sheet. For edge-lifting case, marked atomic-scale friction of the graphene sheet during the nanoscale peeling process is found. During the surface contact, the graphene sheet takes the atomicscale sliding motion. The period of the peeling force curve during the surface contact decreases to the lattice period of the graphite. During the line contact, the graphene sheet also takes the stick-slip sliding motion. These findings indicate the possibility of not only the direct observation of the atomic-scale friction of the graphene sheet at the tip/surface interface but also the identification of the lattice orientation and the edge structure of the graphene sheet.
\end{abstract}

\section{Introduction}

Adhesion and peeling phenomena play important roles in connecting two objects regardless of whether they are inorganic, organic, or biological materials, which contributes to building up microscopic devices. The carbon nanostructures such as, carbon nanotube (CNT) and graphene have recently attracted great interests as the components of the electronic, magnetic, and optical devices. We have so far studied the peeling mechanics of the carbon nanotube (CNT) adsorbed onto the graphite surface both theoretically [1-3] and experimentally $[4,5]$. It is clarified that the transition from the line- to the point-contact between the CNT and the graphite surface occurs during the peeling process [1-5]. The CNT on the sub-microscale has the same size as the spatulae of the microscopic hairs aligned on the gecko foot $[6,7]$. Therefore the study of the peeling process of the nanoscale objects such as, CNT is useful for not only developing the gecko-foot-mimic adhesives [8] but also understanding the elementary process of adhesion.

On the other hand, since the success of its experimental isolation [9], the potential of various applications of the graphene such as, the components of the electronic devices
$[10,11]$ has been discussed by many researchers. There is also a possibility that its adhesion with the substrate is applied to the adhesive tape at nanoscale. Therefore the peeling mechanics of the graphene sheet is very important, which can be regarded as the elementary process of the macroscopic sticky tape such as, the gecko-foot-mimic adhesives [68] or that of the microscopic extension of the crack in the fracture process. In our preliminary experiments, we have already succeeded in peeling the multilayered graphene plate with a thickness of several $\mu \mathrm{m}$ by using atomicforce microscopy tip [12]. Here the two-component epoxy resin adhesive is used to bond the graphene plate to the AFM tip. Here the standard $\mathrm{Si}_{3} \mathrm{~N}_{4}$ tip for the contact AFM experiment is used. The junction formed between the AFM tip and the graphene should be mechanically rigid enough to measure the elasticity of the graphene sheet during the peeling process. The two-component epoxy resin adhesive satisfies the above condition. If the thickness of the peeled graphene plate is reduced, the comparison between the present simulation and the experiment will become possible.

Therefore, in this paper, ahead of experiment, we have theoretically reported the nanoscale peeling behaviors of the monolayer graphene sheet based on the molecular mechanics 
simulation $[13,14]$. The peeling force curve exhibits the nanoscale change of the graphene shape from the surface to the line contact. The center position and the left edge are chosen as the lifting position. In Section 3, the peeling of the monolayer graphene sheet with the armchair edge for lifting the center position is discussed. In Sections 4 and 5, the peeling of the monolayer graphene sheet with the armchairand zigzag-edge for lifting the edge position is discussed, respectively.

\section{Model and Method of Simulation}

In the simulation, a rectangular-shaped monolayer graphene sheet with each side of $38 \AA \times 20-21 \AA$, comprised of 310 carbon atoms, is peeled from the rigid rectangular graphene sheet (which is called, the "graphite surface," hereafter) with each side of $164-165 \AA \times 58 \AA$, comprised of 3536 carbon atoms [Figure 1(a)]. First, both the above graphene sheets are separately optimized by minimizing the covalent bonding energy described by the Tersoff potential energy [15], $V_{\text {cov }}$, using the Polak-Rebiere-type conjugate gradient (CG) method [16]. Here the convergence criterion is set so that the maximum of absolute value of all the forces acting on the movable atoms becomes lower than $10^{-5} \mathrm{eV} / \AA$. Next, the graphene sheet is put and adsorbed onto the graphite surface, so that the $\mathrm{AB}$ stacking registry between the graphene sheet and the graphite surface is satisfied as shown in Figures 1 (b) and 1(c). Here the green-colored six-membered ring at the center position or the outermost left edge of the graphene sheet is assumed to be attached to the AFM tip apex (Figure 1(a)), and then it is gradually moved upward along the $z$ direction, parallel to the [0001] axis, by $0.1 \AA$. For each lifting position of the graphene sheet, $z$, the total energy $V_{\text {total }}=V_{\text {cov }}+V_{\mathrm{vdW}}$ is minimized using the CG method, where $V_{\mathrm{vdW}}$ is the nonbonding vdW interaction described by the modified Lennard-Jones (LJ) potential energy [17, 18], acting between the graphene sheet and the graphite surface. Thus the optimized positions of the movable carbon atoms of the graphene sheet, $(x, y, z)$, the vertical peeling force $F_{z}$, and the lateral sliding forces $F_{x}$ and $F_{y}$, acting on the lifting center, are calculated during the peeling process. In this paper, the graphene sheets with armchair-(Figure 1(b)) and zigzag-edges (Figure 1(c)) are discussed.

\section{Center-Lifting Case of Armchair-Edge Graphene}

When the six-membered ring located at the center position of the monolayer graphene sheet is lifted, the graphene sheet exhibits the characteristic transition of its shape during the peeling process within the $x-z$ plane as illustrated in Figures $2(\mathrm{~A})-2(\mathrm{~J})$, corresponding to Figures $3(\mathrm{~A})-3(\mathrm{~J})$, the vertical force acting on the lifting center position $F_{z}$ plotted as a function of the displacement from the initial position along $z$-direction, $z$. At first the monolayer graphene sheet takes an initial planar structure parallel to the rigid graphite surface (Figure 2(A): $z=0 \AA$ ). Here the surface contact is formed between the graphene sheet and the graphite

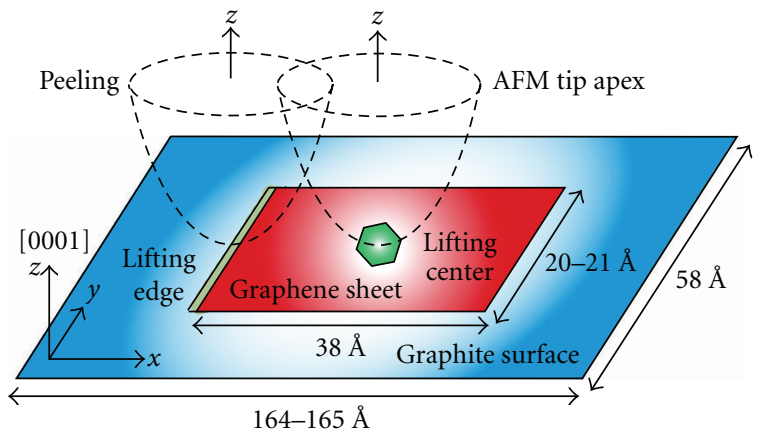

(a)

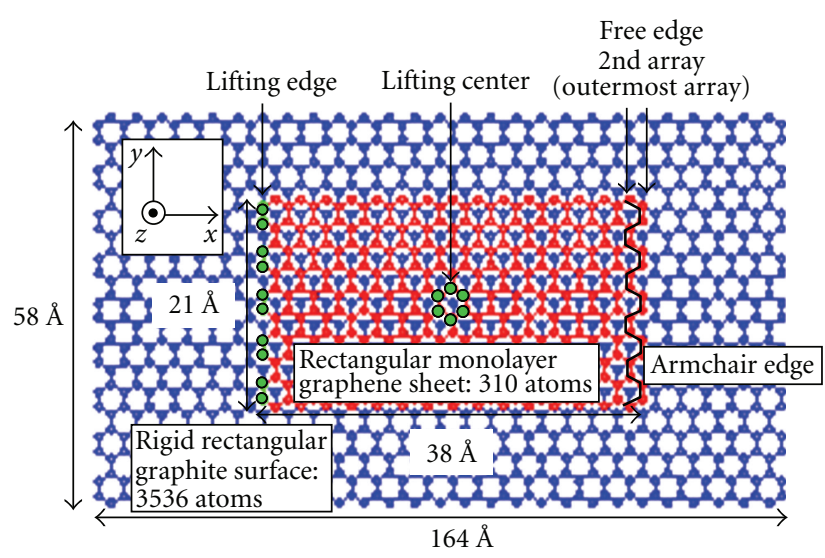

(b)

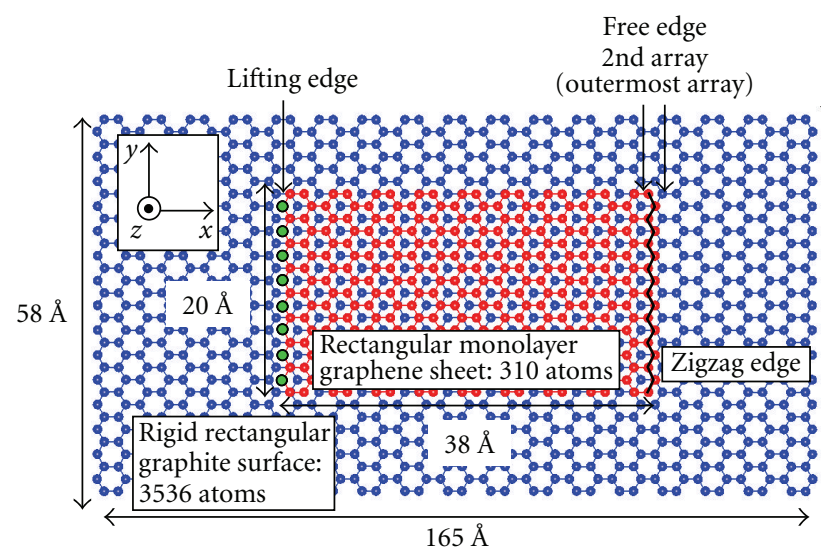

(c)

FIGURE 1: (a) The schematic illustration of the model of the monolayer graphene sheet physically adsorbed onto the rigid graphite surface used in the simulation. The green-colored sixmembered ring at the center position or left edge of the graphene sheet is assumed to be adsorbed onto the atomic force microscopy tip apex indicated by broken lines, and it is moved upward along the $z$ (or [0001]) direction, by $z=0.1 \AA$. Initial AB stacking registry of the red-colored graphene sheet with (b) armchair and (c) zigzag edge adsorbed onto the blue-colored graphite surface within the $x-y$ plane.

surface. The vertical force $F_{z}$ is zero (Figure 3(a)). Just after the beginning of the peeling (Figure 2(B): $z=2.0 \AA$ ), the attractive interaction force takes the minimum value, $-3.1 \mathrm{eV} / \AA$ (Figure 3(B)). 

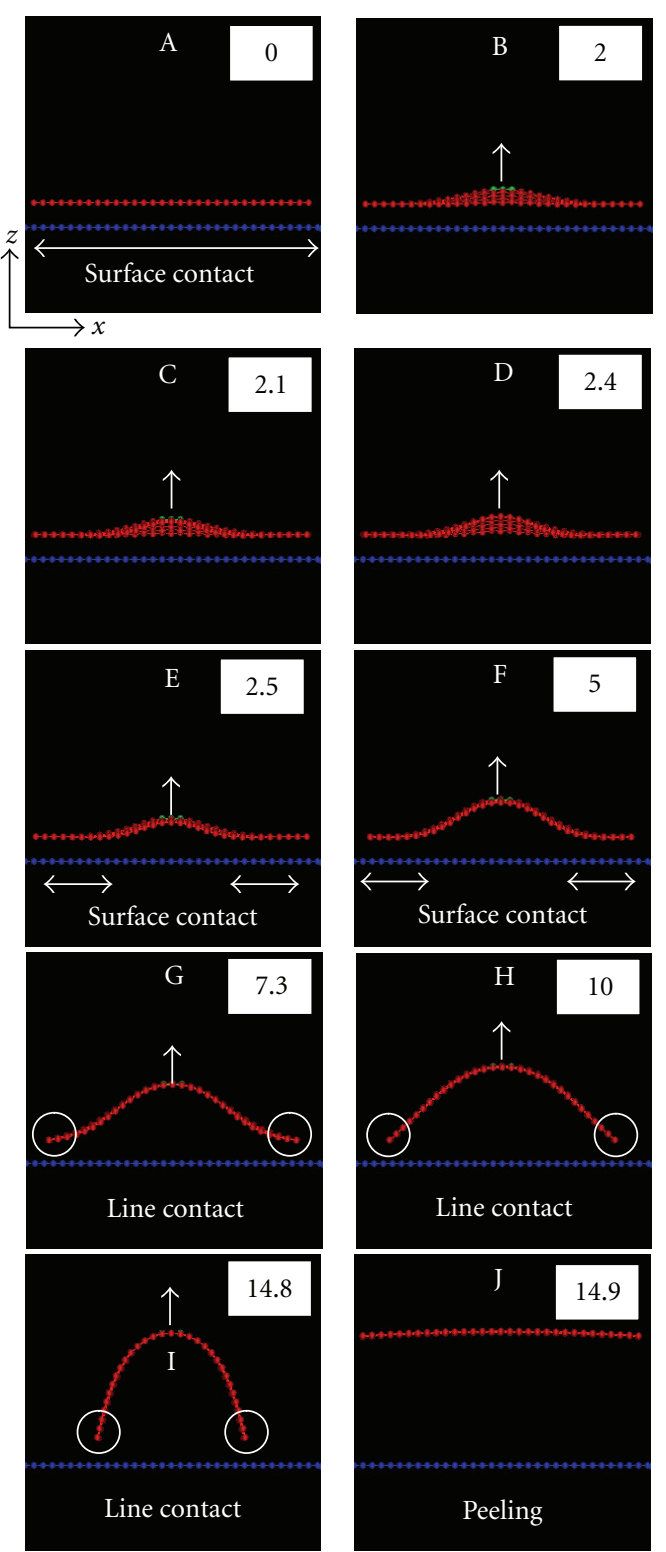

FIgURE 2: The transition of the shape of the monolayer graphene sheet during the peeling process from A to J within the $x-z$ plane. The red-colored graphene sheet and blue-colored graphite surface are shown. The displacement of the lifting center position from the initial position, $z(\AA)$, is indicated on the upper-right positions of each picture.

Between $z=2.0 \AA$ and $2.1 \AA$, the first discrete partial peeling of the graphene occurs (Figures $2(B) \rightarrow 2(C)$ ), which produces the 1st discontinuous jump in the force curve (Figures $3(\mathrm{~B}) \rightarrow 3(\mathrm{C})$ ). The partial peeled area around the lifting center of the graphene is shown in Figures $4(\mathrm{~B}) \rightarrow$ $4(\mathrm{C})$. Then, between $z=2.4 \AA$ and $2.5 \AA$, the second discrete partial peeling of the graphene occurs (Figures $2(D) \rightarrow 2(E)$ ), which produces the 2 nd discontinuous jump in the force curve (Figures $3(\mathrm{D}) \rightarrow 3(\mathrm{E})$ ). The partial peeled area of the graphene is shown in Figures $4(\mathrm{D}) \rightarrow 4(\mathrm{E})$. Which of these two areas is peeled first is expected to be actually the

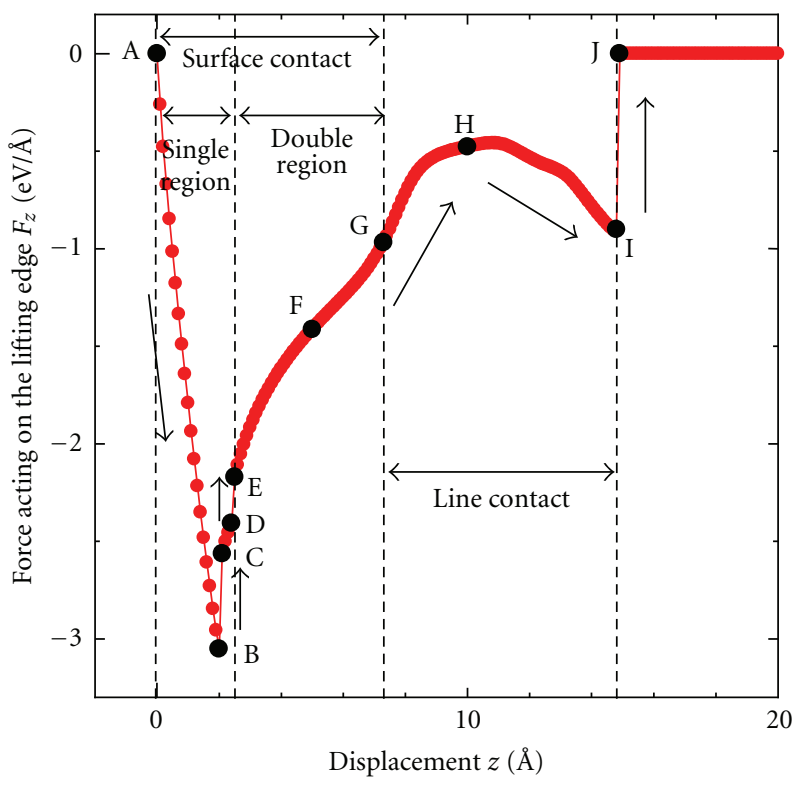

FIGURE 3: The vertical force, $F_{z}$, acting on the center six-membered ring, plotted as a function of the lifting displacement $z$. The positions A-J correspond to those of Figure 2.

stochastic process under the room temperature condition. Now the surface contact region is split into the left and right sections (Figure 2(E)). After the two discrete jumps, $F_{z}$ increases as the peeling proceeds, since the attractive surface contact region gradually decreases (Figure $2(\mathrm{~F}): z=5.0 \AA$ ). Then the surface contact continuously turns into the line contact at $z=7.3 \AA$ (Figure $2(\mathrm{G})$ ). Here the 'line contact' is defined by the following two criteria: (1) The carbon atoms on the left and right outermost arrays of the graphene sheet (Figure 1(b)) receive the repulsive interaction force from the graphite surface. (2) The carbon atoms on the second arrays (Figure 1(b)) next to the outermost arrays receive the attractive interaction force. As illustrated in Figure 5, the average forces acting on one carbon atom on the outermost and the second arrays satisfy the above criteria at $z=7.3 \AA$, which corresponds to Figure $2(\mathrm{G})$.

Once the line contacts are formed between the free edges (outermost arrays) of the peeled graphene sheet and the graphite surface, they clearly slide on the graphite surface as indicated by a circle in Figures $2(\mathrm{G}) \rightarrow 2(\mathrm{H}) \rightarrow 2(\mathrm{I})$ with a rapid increase of the bending of the graphene sheet. Within $x-y$ plane, the right outermost array of the graphene sheet slides nearly straightforward along $-x$ direction, not so sensitive to the lattice structure of the surface as illustrated in Figures 6(a) and 6(b), which show the trajectories of the two carbon atoms on the right outermost array illustrated in Figure 1(b). The sliding of the outermost arrays during $\mathrm{G}$ and I appears much more clearly than that during $\mathrm{A}$ and G. During $\mathrm{H}$ and $\mathrm{I}$, the decrease of $F_{z}$ (Figures $3(\mathrm{H})$ and $3(\mathrm{I})$ ) can be explained by the decrease of the repulsive force acting on the carbon atoms on the left and right edges of the graphene sheet as shown in Figure 5, that is to say, the relative increase of the effect of the attractive interaction force. 

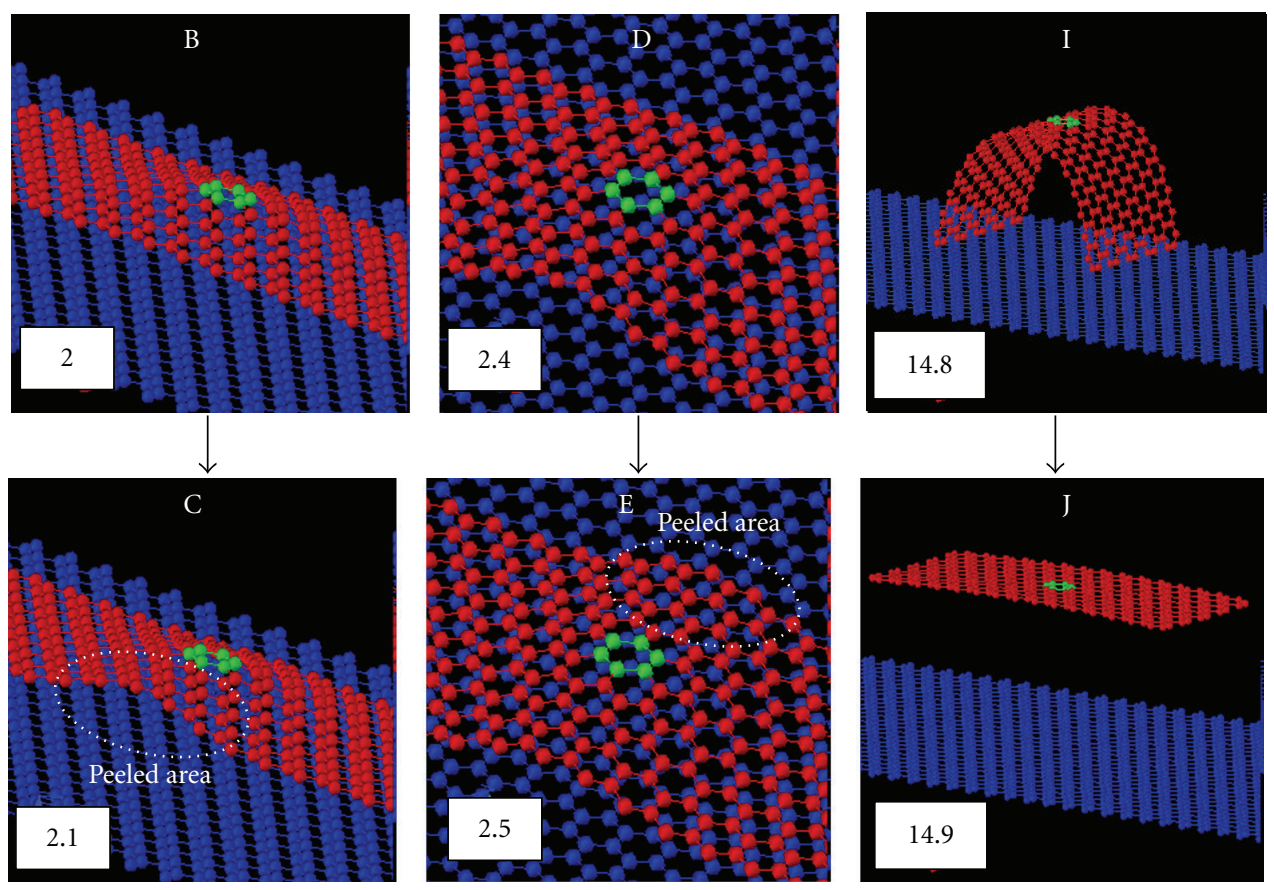

FIgURE 4: The atomic structures of the graphene sheet just before and after the discrete change, $\mathrm{B} \rightarrow \mathrm{C}, \mathrm{D} \rightarrow \mathrm{E}$, and $\mathrm{I} \rightarrow \mathrm{J}$. The regions surrounded by dotted ellipses show the partial peeled areas.

When the bending of the graphene sheet becomes larger than a certain range, both the left and right line contacts break and the graphene sheet is completely peeled from the surface (Figures $2(\mathrm{I}) \rightarrow 2(\mathrm{~J}): z=14.8 \AA \rightarrow 14.9 \AA$ ), which produces the 3rd discontinuous jump in the force curve (Figures $3(\mathrm{I}) \rightarrow 3(\mathrm{~J})$ ). As illustrated in Figures $2(\mathrm{I}) \rightarrow 2(\mathrm{~J})$ and Figures $4(\mathrm{I}) \rightarrow 4(\mathrm{~J})$, the graphene sheet exhibits the transition from the arched shape to the planer shape.

Thus the vertical peeling force $F_{z}$ exhibits the characteristic shape as shown in Figure 3, which reflects the transition from the surface to the line contact between the graphene sheet and the graphite surface. On the other hand, the lateral sliding force $F_{x}$ is zero due to the structural symmetry of the system. However, the lateral sliding force $F_{y}$ shows a finite value with an oscillation whose period and amplitude decrease as $z$ increases. This oscillation of Figure 7 reflects the trajectory of the graphene edges illustrated in Figure 6 at the graphene-substrate interface during the peeling process. The maximum lateral force $F_{y} \simeq 0.1 \mathrm{eV} / \AA$ which is only about $3 \%$ of the absolute value of the maximum adhesion force $\left|F_{z}\right|=3.1 \mathrm{eV} / \AA$.

\section{Edge-Lifting Case of Armchair-Edge Graphene}

4.1. Nanoscale Peeling along Vertical Direction. When the left edge of the monolayer graphene sheet with armchair edge (Figure 1(b)) is lifted, the shape of the graphene sheet markedly changes during the peeling process within the $x-z$ plane as illustrated in Figures $8(\mathrm{~A})-8(\mathrm{~J})$, corresponding to
Figures 9(a)A-9(a)J, the vertical force acting on the lifting edge $F_{z}$ plotted as a function of the edge height $z$.

4.1.1. Surface-Contact Region. At first the monolayer graphene sheet takes an initial planar structure parallel to the rigid graphite surface (Figure $8(\mathrm{~A}): z=0 \AA$ ), and the vertical force $F_{z}$ is zero (Figure $9(\mathrm{a}) \mathrm{A}$ ), which means the graphene sheet completely takes the surface contact with the graphite surface. Just after the start of the peeling (Figure 8(B): $z=1.1 \AA$ ), the attractive interaction force $\left|F_{z}\right|$ becomes the maximum, $0.74 \mathrm{eV} / \AA$ (Figure 9(a)B). After that the surface contact area gradually decreases as the peeling proceeds (Figures $8(\mathrm{C})-8(\mathrm{E})$ ), where Figure 9 (a) exhibits the atomic-scale zigzag structures (Figures $9(\mathrm{a}) \mathrm{C}-\mathrm{E}$ ), which will be explained in the Section 4.2.

4.1.2. Line-Contact Region. After the surface contact vanishes, the line contact appears (Figure $8(\mathrm{~F})$ ). Here the "line contact" is defined by the following two criteria similar to the case of the center-lifting peeling. (1) The outermost array of the free edge of the graphene sheet (Figure 1(b)) receives the averaged repulsive interaction force per one carbon atom from the graphite surface. (2) The second array next to the outermost array (Figure 1(b)) receives the averaged attractive interaction force per one carbon atom. As illustrated in Figure 9(b), the edge height $z=31.3 \AA$ satisfies the above criteria (Figure $8(\mathrm{~F})$ ). Here the free edge adsorbed onto the graphite surface is nearly fixed even if the left edge is lifted (Figure $8(\mathrm{~F}) \rightarrow$ Figure $8(\mathrm{G}): z=33.9 \AA)$. As a result, the inplane bending of the graphene sheet markedly decreases, 


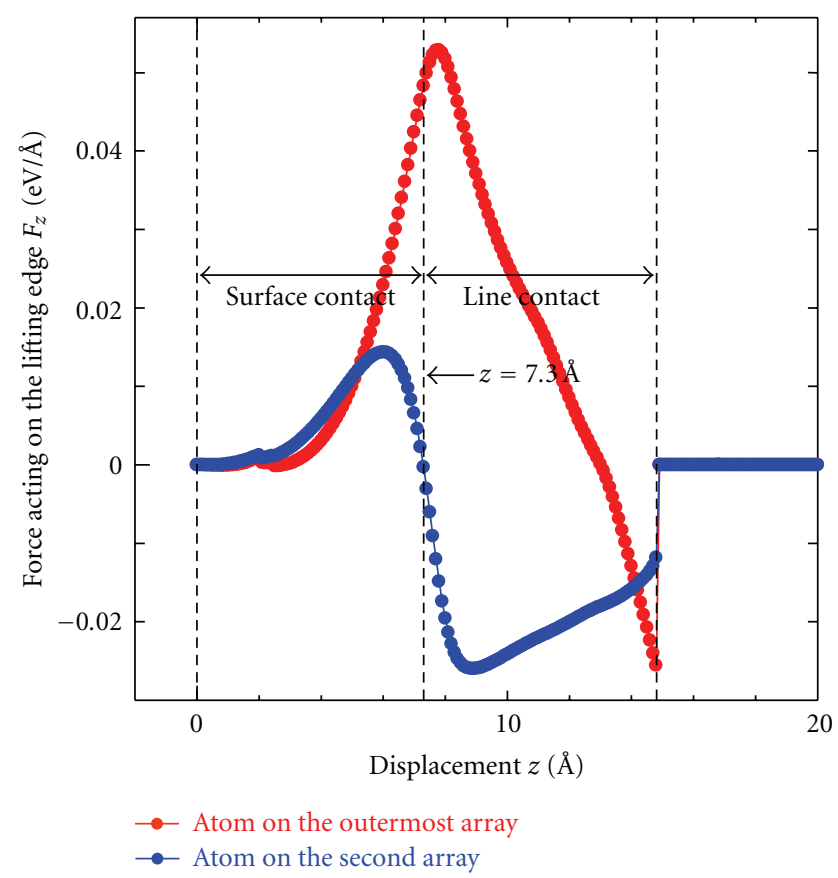

FIGURE 5: The averaged forces acting on one atom on the left and right outermost arrays (red-colored) and those on the left and right second arrays (blue-colored), as a function of the displacement of the lifting center position from the initial position, $z(\AA)$.

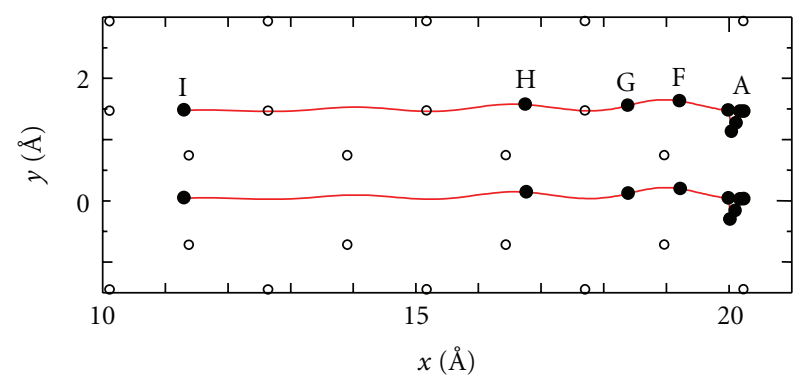

(a)

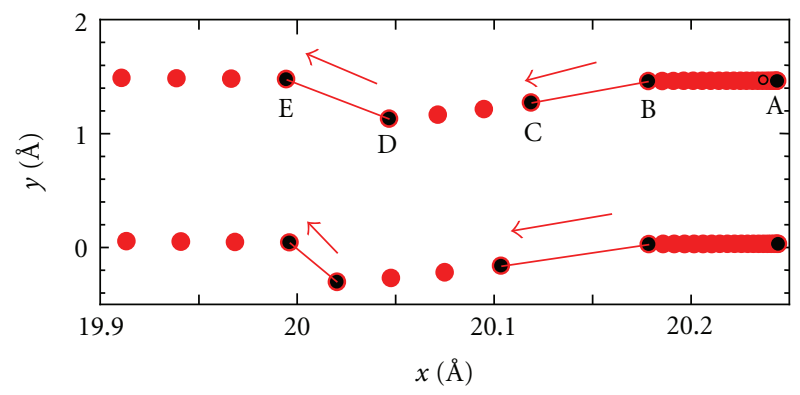

(b)

FIgure 6: The trajectory of the two carbon atoms on the right free edge (outermost array) indicated by white circles in Figure 1(b). (a) The whole trajectory $\mathrm{A} \rightarrow \mathrm{I}$, and (b) the part of the trajectory $\mathrm{A} \rightarrow \mathrm{E}$, including the discrete jumps, $\mathrm{B} \rightarrow \mathrm{C}$ and $\mathrm{D} \rightarrow \mathrm{E}$, are indicated. White circles mean carbon atoms of the graphite surface. The indices A-I correspond to those in Figure 2.

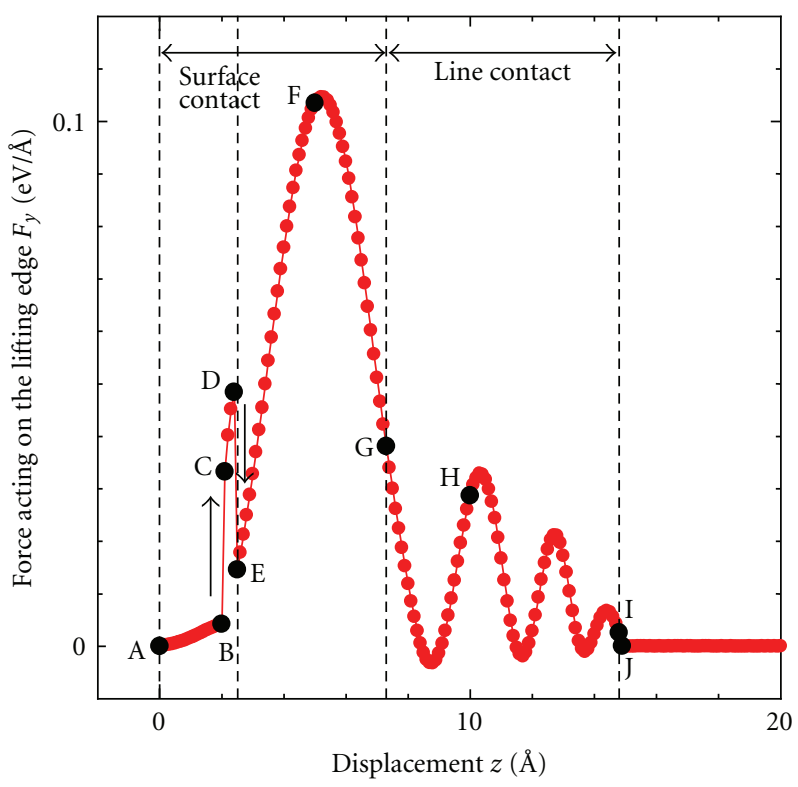

FIGURE 7: The lateral force, $F_{y}$, acting on the center six-membered ring, plotted as a function of the displacement $z$. The positions A-J correspond to those of Figure 2.

and $\left|F_{z}\right|$ decreases to zero (Figures $9(\mathrm{a}) \mathrm{F}-\mathrm{G}$ ). After that the free edge slides on the surface until the graphene sheet takes planar structure within the $y-z$ plane (Figure $8(\mathrm{H}): z=$ $37.9 \AA$ ), where $\left|F_{z}\right|$ increases again (Figure $9(\mathrm{a}) \mathrm{H}$ ).

4.1.3. Toward Complete Peeling. Then the graphene sheet is continuously moved upward (Figure 8(I): $z=38.0 \AA \rightarrow$ Figure $8(\mathrm{~J}): z=38.4 \AA$ ) and is completely peeled from the surface. It is noted that the line contact clearly vanishes at $z=38.0 \AA$ (Figure 9(b)I). The attractive interaction force $\left|F_{z}\right|$ increases to take the maximum value (Figures $9(\mathrm{a}) \mathrm{I} \rightarrow \mathrm{J}$ ), and then it gradually decreases to zero toward the complete peeling.

4.2. Atomic-Scale Sliding within Lateral Plane. Figure 9(a) shows the atomic-scale zigzag structures within the surfaceand line-contact regions, which can be explained by the following atomic-scale sliding motions of the graphene sheet within the $x-y$ plane.

4.2.1. Surface-Contact Region. During the surface contact region between $\mathrm{C}$ and $\mathrm{E}$ in Figure 9(a), $z-F_{z}$ curve takes the atomic-scale zigzag structures from I to VII. The zigzag behaviors exhibit the transition from the continuous (Figures 10(a)I-II) to the sawtooth shapes (Figures 10(a)III-VII). First Figures 10 (a) $1 \rightarrow 2 \rightarrow 3 \rightarrow 4 \rightarrow 5$ correspond to Figures 10 (b) $1 \rightarrow 2 \rightarrow 3 \rightarrow 4 \rightarrow 5$, which show that the graphene sheet continuously slides passing over the nearest neighboring $\mathrm{AB}$ stacking sites with the graphite surface. The trajectories of the graphene sheet exhibit the continuous zigzag paths as shown in Figure 10(b)5. Next Figures 10(a) $6 \rightarrow 7 \rightarrow 8 \rightarrow$ $9 \rightarrow 10$ correspond to Figures 10 (c) $6 \rightarrow 7 \rightarrow 8 \rightarrow 9 \rightarrow 10$ which show that the graphene sheet takes the zigzag stick-slip 

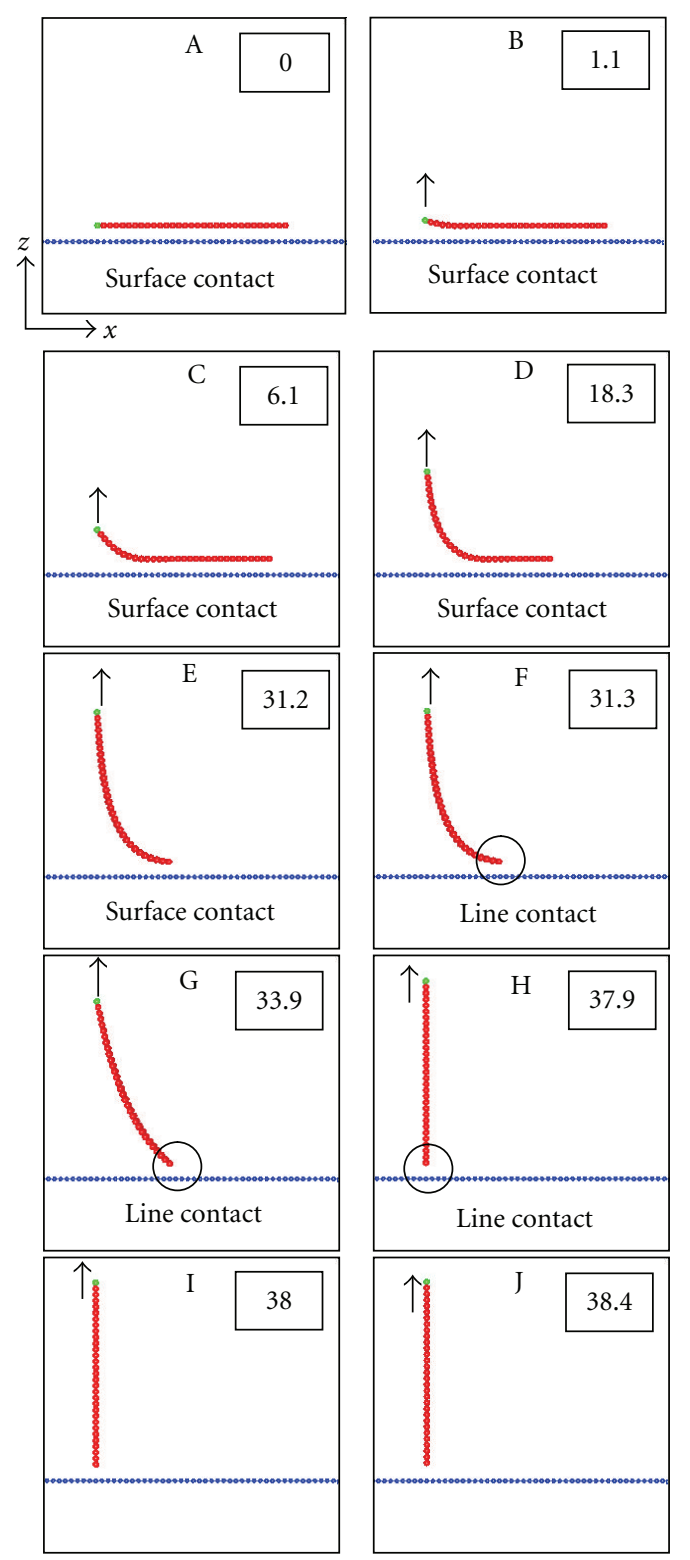

FIGURE 8: The transition of the shape of the monolayer graphene sheet with free edge of armchair-type during the peeling process from A to J within the $x-z$ plane. The red-colored graphene sheet and blue-colored graphite surface are shown. The height of the lifting left edge $z(\AA)$ is indicated on the upper-right positions of each picture.

motions between the nearest neighboring $\mathrm{AB}$ stacking sites. Just before the slip, the graphene sheet deviates quite a little from the AB-stacking site (Figure 10(c) $6: z=17.3 \AA$ ). Then it discretely jumps or slips to the neighboring $\mathrm{AB}$ stacking site (Figure 10(c)7: $z=17.4 \AA$ ). As the peeling proceeds, the graphene sheet continuously slides quite a little (Figure 10(c)8: $z=18.2 \AA$ ), then it discretely slips again to the neighboring $\mathrm{AB}$ stacking site (Figure 10(c)9: $z=18.3 \AA$ ). After that the graphene sheet continuously slides quite a little again (Figure 10(c)10: $z=20.1 \AA$ ) until the next slip toward the neighboring $\mathrm{AB}$ stacking site occurs. As a result

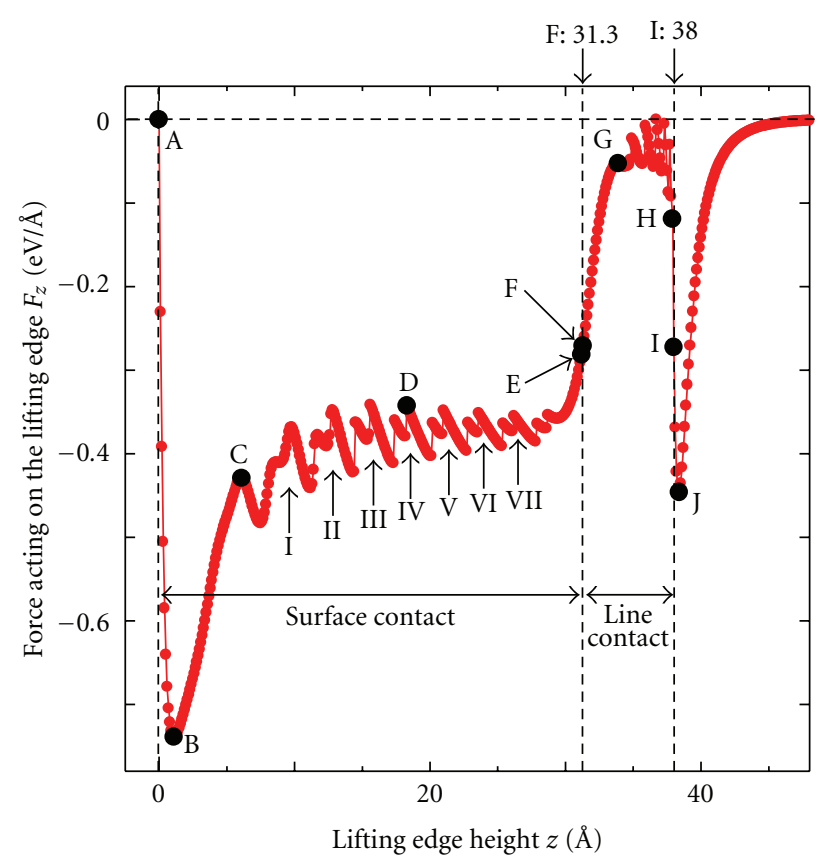

(a)

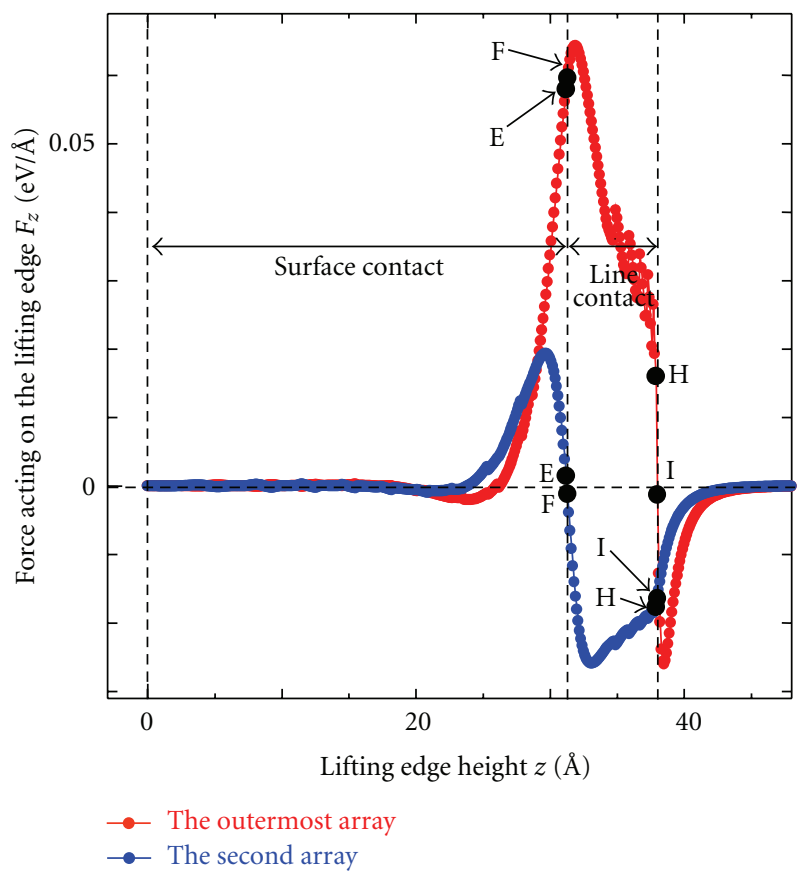

(b)

Figure 9: (a) The vertical force, $F_{z}$, acting on the lifting edge, plotted as a function of the lifting edge height $z$ for the graphene with armchair-type free edge. The indices A-J correspond to those of Figure 8. (b) The red-colored averaged force per one atom acting on the outermost array, and the blue-colored one acting on the second array, as a function of the lifting edge height $z(\AA)$. The indices E, F, H, and I correspond to those of Figures 8 and 9 (a).

the trajectories of the graphene sheet exhibit the discrete zigzag paths connecting the nearest neighboring $\mathrm{AB}$-stacking sites as shown in Figure 10(c)10. The period of the zigzag 


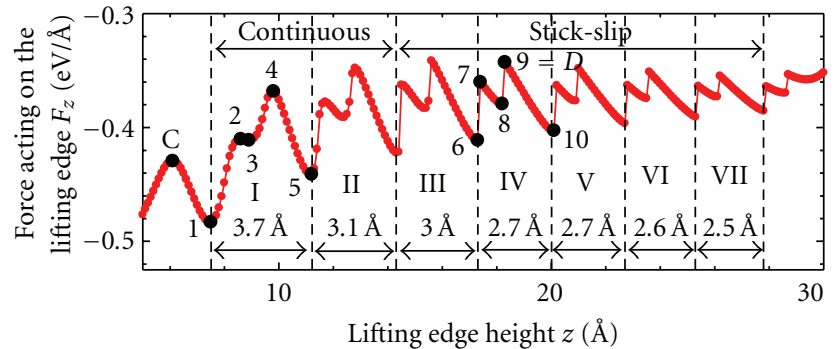

(a)

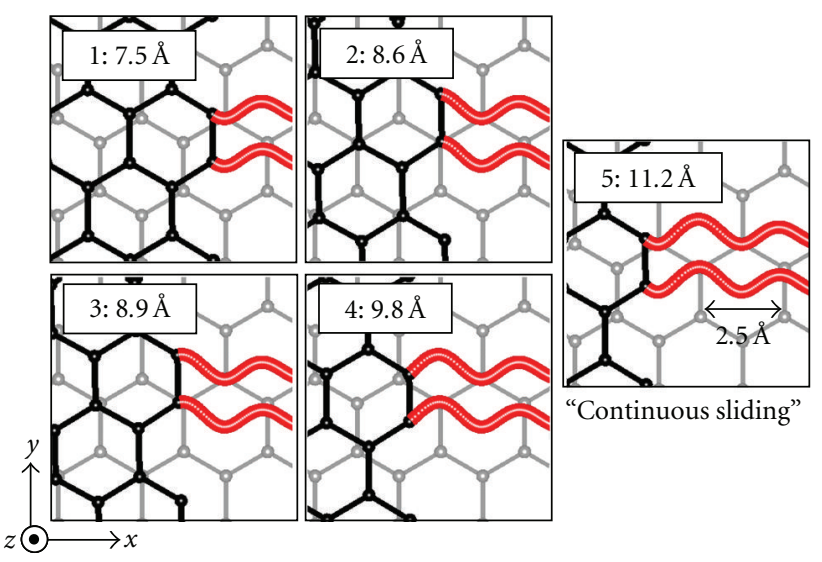

(b)

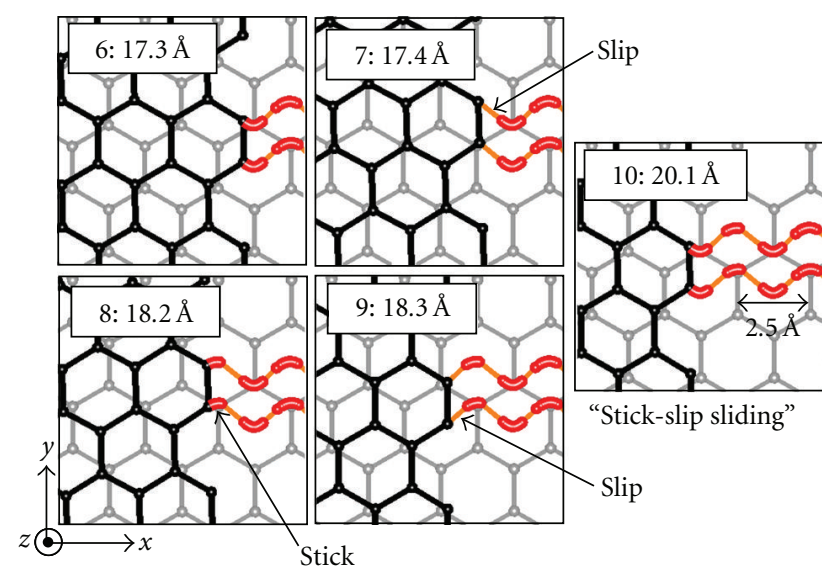

(c)

FIGURE 10: (a) Enlargement of part of the $z-F_{z}$ curve (Figure 9(a)) corresponding to the continuous and stick-slip process during the surface contact. (b) The trajectories of the two carbon atoms on the free edge from 1 to 5 indicated in (a). (c) The trajectories of the two carbon atoms on the free edge from 6 to 10 indicated in (a).

behavior of the $F_{z}$ curve decreases from $3.7 \AA$ to $2.5 \AA$ as shown in Figure $10(\mathrm{a})$ as the peeling proceeds. The lattice spacing of the graphite surface, $2.5 \AA$, appears in the peeling force curve particularly for the stick-slip region.

4.2.2. Line-Contact Region. During the line contact region between $\mathrm{G}$ and $\mathrm{H}$ in Figure 9(a), $z-F_{z}$ curve takes another atomic-scale zigzag structures as shown in Figure 11(a). One of the zigzag behaviors in the force curve (Figures 11(a) $1 \rightarrow$

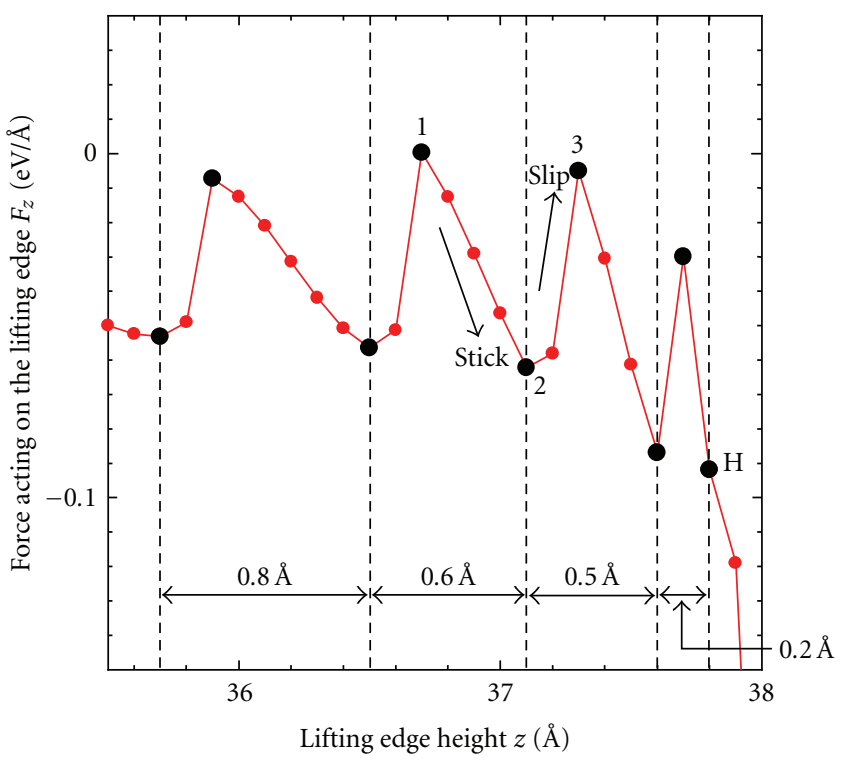

(a)

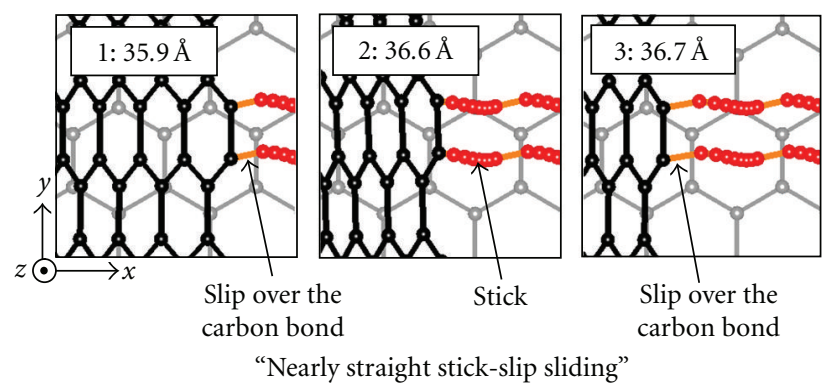

(b)

FIGURE 11: (a) Enlargement of part of the $z-F_{z}$ curve (Figure 9(a)) corresponding to the nearly straight stick-slip region during the line contact. (b) The trajectories of the two carbon atoms on the free edge from 1 to 3 indicated in (a).

$2 \rightarrow 3$ ) corresponds to the stick-slip sliding motion of the graphene sheet (Figures $11(\mathrm{~b}) 1 \rightarrow 2 \rightarrow 3$ ). Here the free edge of the graphene sheet slides with nearly the straight stick-slip motions. One of the carbon atoms on the free edge passes over the carbon-carbon bonds as shown in Figures 11(b)1 and 3.

\section{Edge-Lifting Case of Zigzag-Edge Graphene}

Recently it has been reported that the edge structure of the graphene sheet plays quite an important role in electronic, magnetic, and optical properties of graphene, which can be also expected to give influences on the mechanical properties such as, the peeling process. Therefore, in this section, the peeling process of the graphene sheet with zigzag edge is discussed. In the simulation, the model obtained by rotating Figure $1(\mathrm{~b})$ by $30^{\circ}$ is used (Figure $1(\mathrm{c})$ ), and the left zigzag edge is lifted to simulate the peeling process, while the right free edge is zigzag type. As a result, the nanoscale peeling process within the $x-z$ plane and the global shape of the 


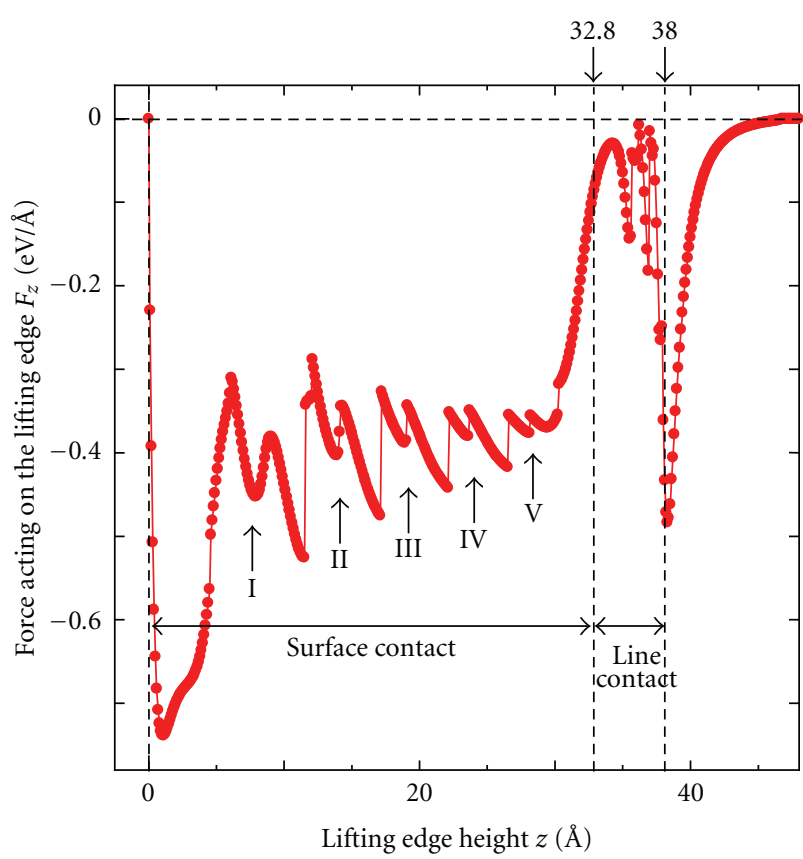

FIGURE 12: The vertical force, $F_{z}$, acting on the lifting edge, plotted as a function of the lifting edge height $z$ for the graphene with zigzag-type free edge.

force curve (Figure 12) is similar to that of Figures 8 and 9, respectively. The qualitative tendency of the decrease of the period and amplitude of the force curve (Figure 12(b)I-IV) is similar to that for Figure 9(a)I-VII. However, the details of the atomic-scale mechanics of the zigzag edge are clearly different from those of the armchair edge as follows.

During the surface contact, the graphene sheet first takes zigzag (Figures 13(b)1-6) and then straight stickslip motions (Figures 13(c)7-11), passing over the nearest neighboring AB-stacking site along [10 $\overline{1} 0]$ direction. It is noted to avoid AA-stacking registry, the graphene sheet takes zigzag slip toward the nearest neighboring $\mathrm{AB}$-stacking site as shown in Figure 13(b) $1 \rightarrow 2$, although it takes straight slip as shown in Figure $13(\mathrm{c}) 7 \rightarrow 8$. The minimum period of the force curve of $4.4 \AA$ (Figure $13(\mathrm{a}) \mathrm{IV}$ ) reflects the lattice period of the graphite surface along the [10 $\overline{1} 0]$ direction, while $2.5 \AA$ for the armchair-type edge (Figure 10(a)VII) reflects that along the $[12 \overline{3} 0]$ direction. Thus the edge structure gives the marked effects on the atomic-scale dynamics depending on the lattice orientation of the surface.

During the line contact, the difference between the armchair- and zigzag-type edge is enhanced. Figure 14(a) reflects the zigzag stick-slip motion of the graphene sheet (Figures 14(b)1-9) unlike nearly the straight stick-slip motion (Figures 10(b)1-3). Important point of the linecontact sliding is that each carbon atom on the free edge takes stick-slip motion between the nearest neighboring sixmembered rings. When each atom is located on the hollow site of the six-membered ring, the graphene sheet does not deform along the $y$ direction (Figures 14 (b) 1, 4-5, and 89). However, when each atom is located a little far from

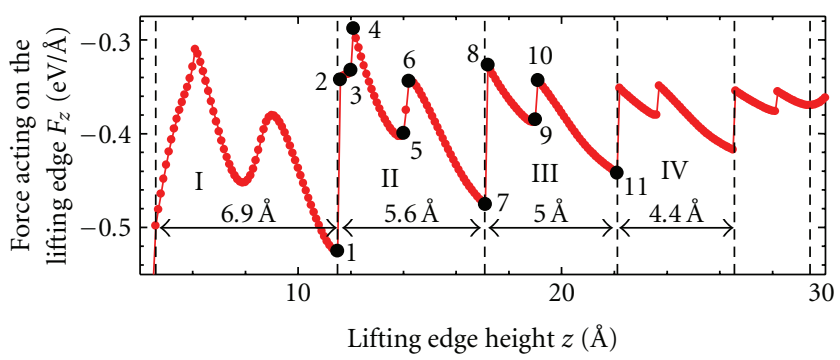

(a)
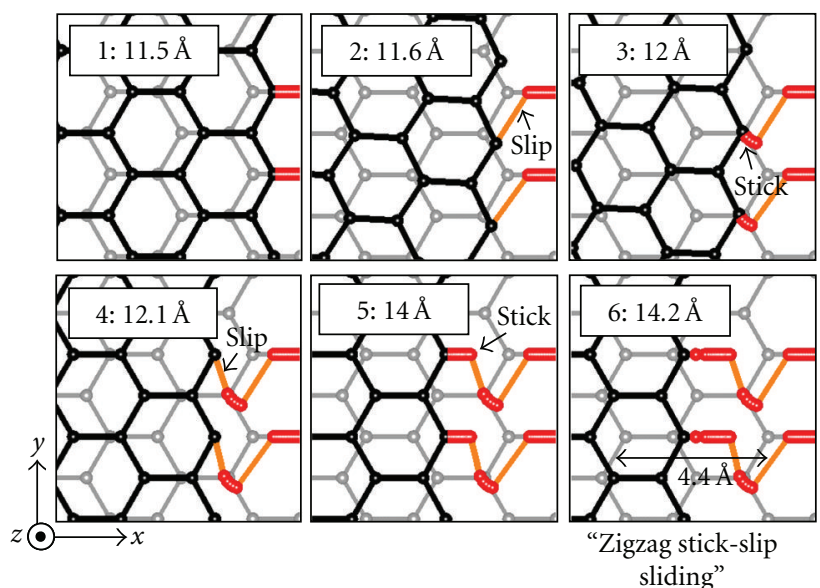

(b)

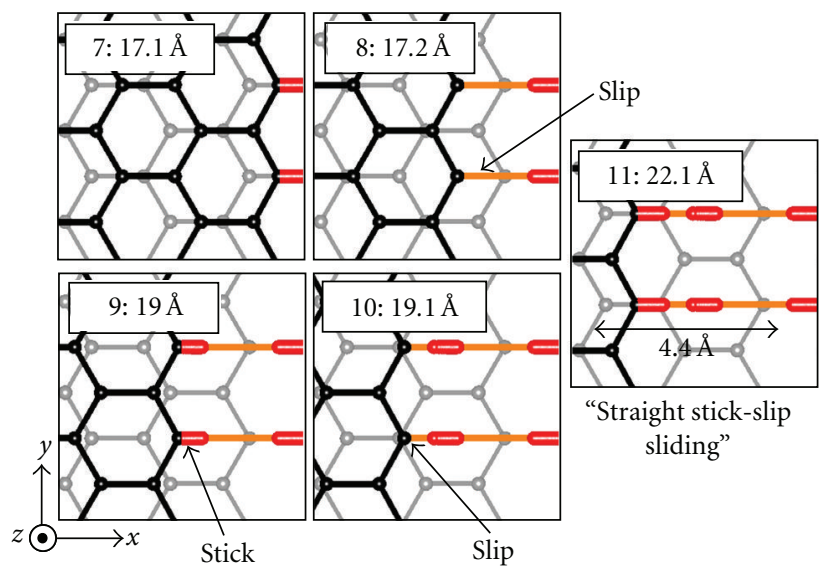

(c)

Figure 13: (a) Enlargement of part of the $z-F_{z}$ curve (Figure 12(b)) corresponding to the zigzag and straight stick-slip process during the surface contact. (b) The trajectories of the two carbon atoms on the free edge from 1 to 6 indicated in (a). (c) The trajectories of the two carbon atoms on the free edge from 7 to 11 indicated in (a).

the hollow site or near the carbon bond, the graphene sheet bends toward the $y$ direction to decrease the total interaction energy (Figures 14(b)2-3 and 6-7). Thus, in the case of the zigzag-type edge, collective motion of the single carbon "atom" on the free edge nearly dominates the graphene mechanics together with its deformation. On the other hand, for the armchair-type edge, collective motion of the single carbon "bond" is dominant. 


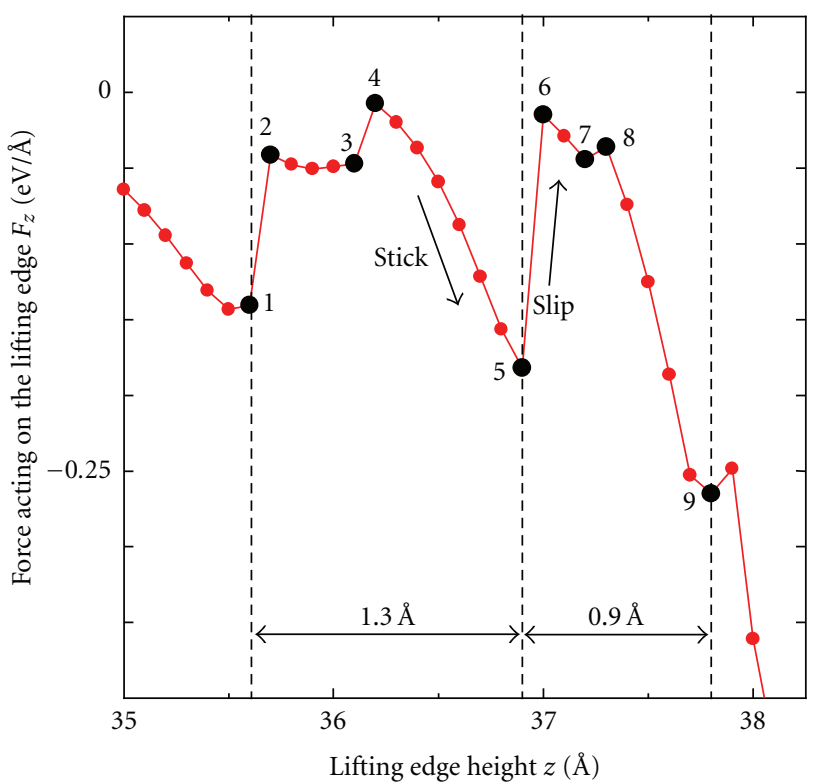

(a)
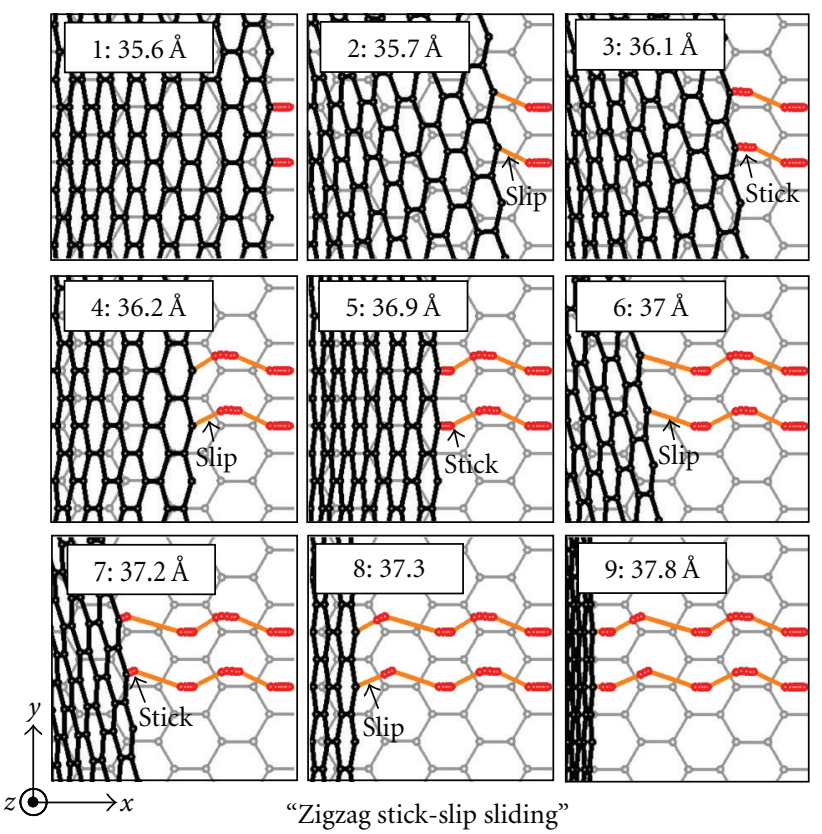

(b)

Figure 14: (a) Enlargement of part of the $z-F_{z}$ curve (Figure 12(b)) corresponding to the zigzag stick-slip process during the line contact. (b) The trajectories of the two carbon atoms on the free edge from 1 to 9 indicated in (a).

\section{Discussions and Conclusions}

In this work molecular mechanics study of the nanoscale peeling of the monolayer graphene sheet has been performed. The peeling force curve clearly exhibits the change of the graphene shape from the surface- to the line-contact.

In Section 3, the peeling of the monolayer graphene sheet with the armchair edge for lifting the center position is discussed. It is noted that the maximum lateral sliding force
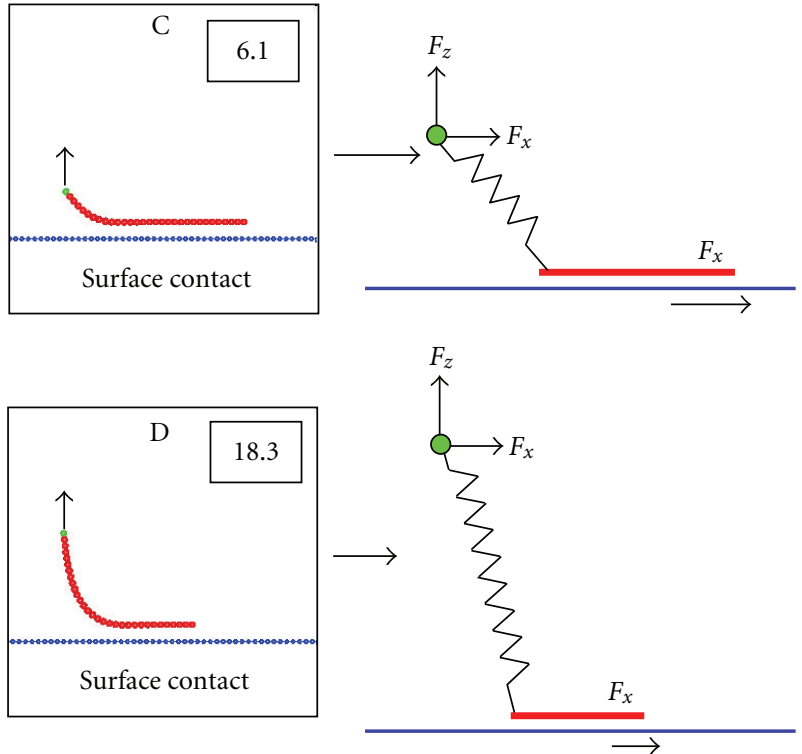

(a)
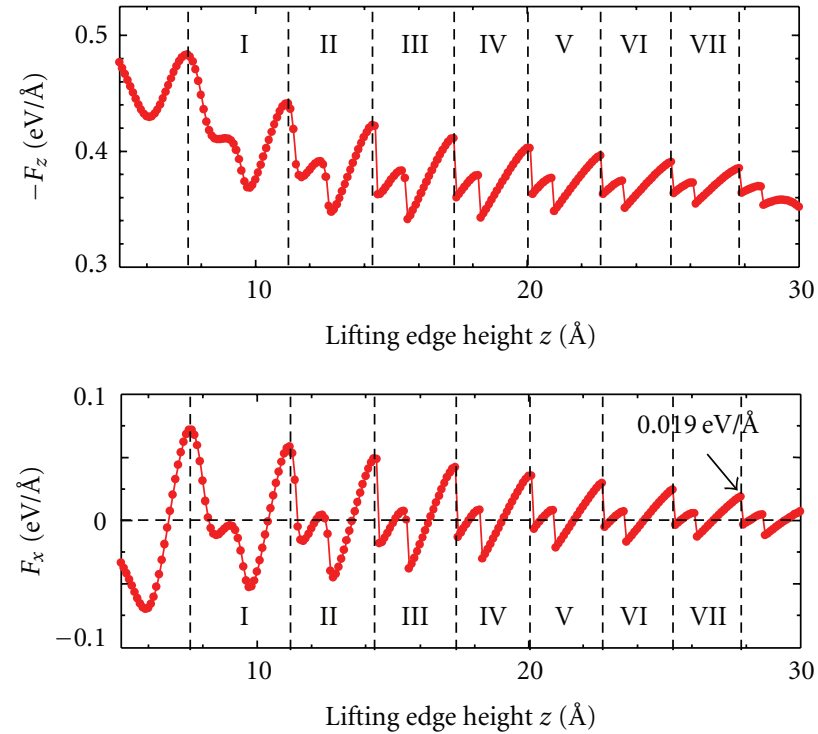

(b)

Figure 15: (a) Schematic illustration of the increase of the peeled area and the decrease of the surface contact area from C $(z=6.1 \AA)$ to $\mathrm{D}(z=18.3 \AA)$ for the graphene sheet with armchair-type free edge. (b) $-F_{z}$ and $F_{x}$ plotted as a function of the edge height $z$, show qualitatively the same behavior to each other for the graphene sheet with armchair-type free edge.

$F_{y} \simeq 0.1 \mathrm{eV} / \AA$ is only about $3 \%$ of the absolute value of the maximum adhesion force $\left|F_{z}\right| \simeq 3.1 \mathrm{eV} / \AA$. This small sliding force $F_{y}$ is derived from the superlubricity at the interface between the graphene sheet and the graphite surface [13] and atomic-scale wear [19]. There is a possibility that such anisotropy between the vertical force $F_{z}$ and the lateral sliding force $F_{y}$ can be applied to the adhesives, which can be strongly adhered to the substrate but can easily slide on it. Our AFM measurement exhibits that the maximum pull-off 


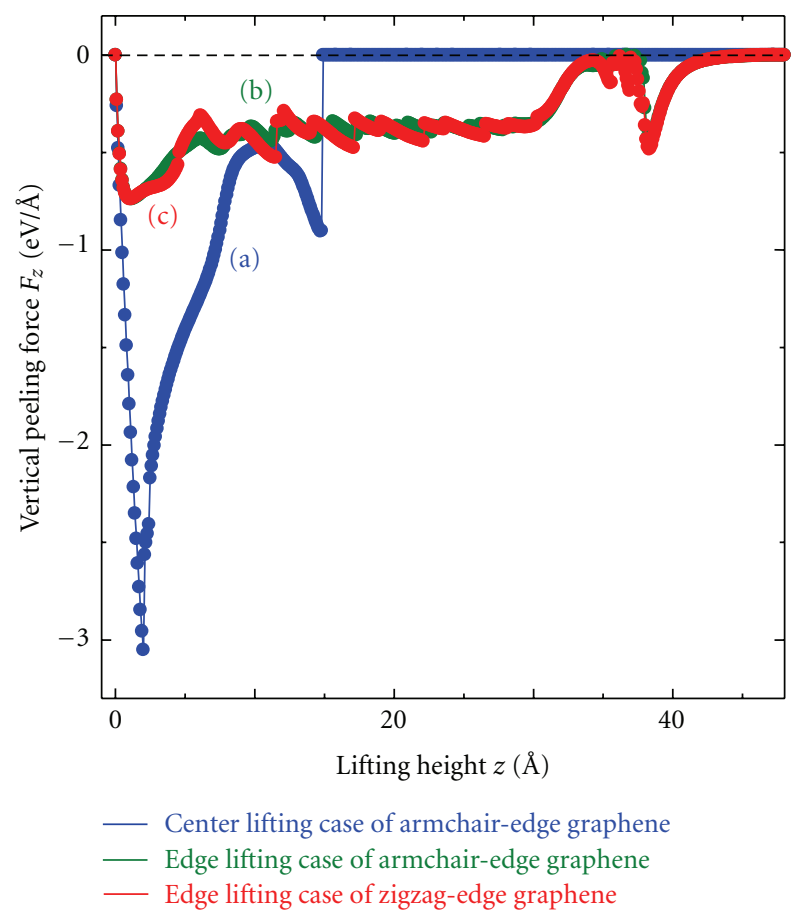

Figure 16: Comparison of the vertical forces, $F_{z}$, among (a) centerlifting case of the armchair-edge graphene sheet, (b) edge-lifting case of the armchair-edge graphene sheet, and (c) edge-lifting case of the zigzag-edge graphene sheet.

force is about several hundreds of $\mathrm{nN}$, which is clearly much larger than the binding force, $3 \mathrm{eV} / \AA \bumpeq 4.8 \mathrm{nN}$, assumed in our simulation.

In Sections 4 and 5, the peeling of the monolayer graphene sheet with the armchair- and zigzag-edge for lifting the edge position is discussed, respectively. The atomicscale sliding motion of the monolayer graphene sheet during the peeling process is found. For the graphene sheet with armchair edge, the transition from the continuous to the stick-slip motion of the graphene sheet is found, which can be explained as follows. The peeling process induces the increase of the peeled area of the graphene sheet and the decrease of the surface contact area. Considering that the peeled area of the graphene sheet acts as an effective spring as shown in Figure 15, the increase of the peeled area makes the effective spring softer, and the decrease of the surface contact area decreases the energy barrier to slide the graphene sheet. Finally the peeling process induces the transition from the continuous to the stick-slip sliding motion of the graphene sheet, together with the decrease of the period and amplitude of the $z-F_{z}$ curve. An important point is that the period of the peeling force curve for the armchair-edge graphene for the surface contact region corresponds to the lattice spacing of the graphite surface along $[12 \overline{3} 0]$ direction, $2.5 \AA$. On the other hand, for the zigzag-edge graphene, the period becomes the lattice spacing along [10 $\overline{1} 0]$ direction, $4.4 \AA$. This means the sliding length of the graphene sheet along $x$ direction becomes nearly equal to the peeled length along $z$ direction. The zigzag

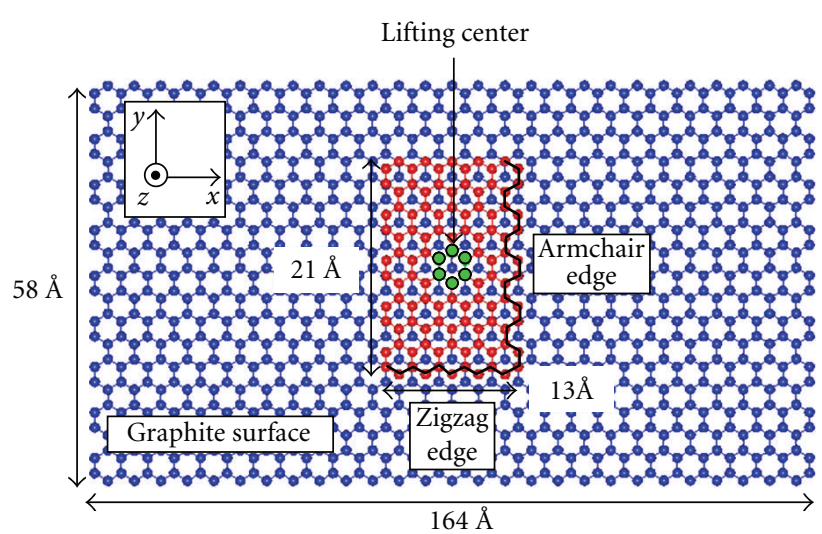

(a)

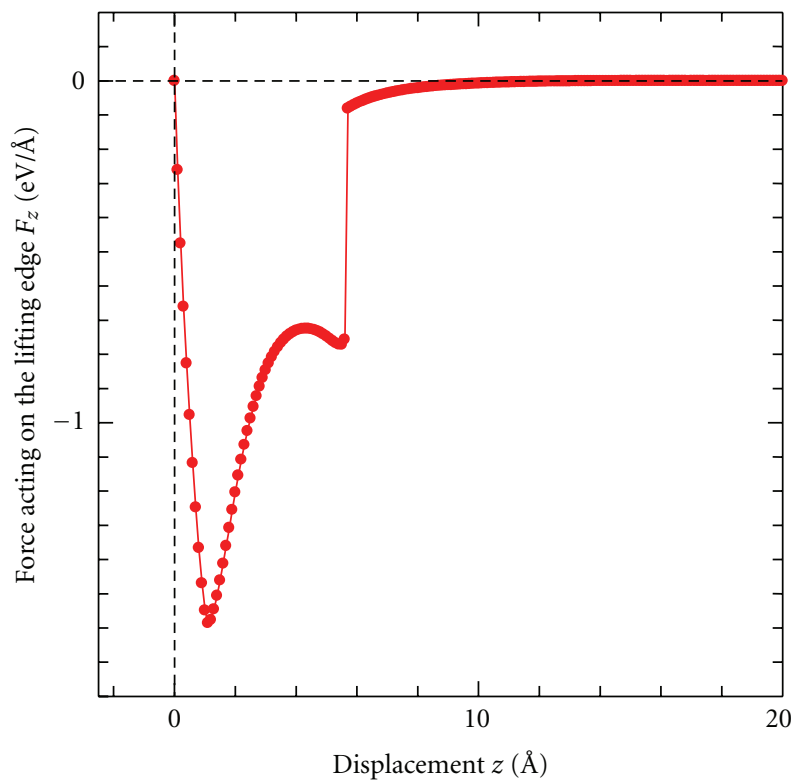

(b)

Figure 17: (a) The model of the red-colored monolayer graphene sheet physically adsorbed onto the blue-colored rigid graphite surface within the $x-y$ plane. The green-colored six-membered ring at the center position is moved upward along the $z$ (or [0001]) direction, by $z=0.1 \AA$. Initial $\mathrm{AB}$ stacking registry of the redcolored graphene sheet with the blue-colored graphite surface is assumed. (b) The vertical force, $F_{z}$, acting on the center sixmembered ring, plotted as a function of the lifting displacement $z$.

structures of the peeling force curve with the same period of about several $\AA$ have been also observed by our preliminary experiments using the multilayered graphene, which will be reported elsewhere [12]. Of course, if the number of the peeled graphene sheets is reduced, the direct comparison between the present simulation and the experiment will become possible.

As a result, the center-lifting case requires the largest attractive peeling force, $-3.1 \mathrm{eV} / \AA$, in order to peel the graphene sheet as shown in Figure 16(a). On the other hand, the edge-lifting case requires only $-0.74 \mathrm{eV} / \AA$, about $20 \%$ of that for the center-lifting case as shown in Figures 16(b) and 
16(c). The edge structures give little influences on the basic features of the force curve. However, the sliding direction and the edge structure clearly give marked influences on the surface- and line-contact regions, respectively.

Another important point is that the behavior of the lateral force curve $\left(F_{x}(z)\right)$ is qualitatively the same as that of the vertical force curve $\left(F_{z}(z)\right)$ during the surface contact as shown in Figure 9(b). Therefore, it can be said that the peeling force curve, $F_{z}(z)$, directly reflects the atomicscale friction force, $F_{x}(z)$, which decreases to $0.019 \mathrm{eV} / \AA \bumpeq$ $30 \mathrm{pN}$ for $z=27.8 \AA$ (Figure 9(b)). This ultralow friction force, $F_{x}$, is derived from the superlubricity at the interface between the graphene sheet and the graphite surface [1921]. Furthermore, effect of the edge structure on the peeling process is clarified by comparison of the free edge between the armchair- and zigzag-types. As mentioned above, the atomic-scale structure of the force curve during the surface contact reflects the lattice spacing of the graphite surface. So the minimum period of the atomic-scale structure of the force curve can tell us the atomic-scale lattice orientation and structure of the free edge of graphene. Such information can be used for the control of the electronic properties of the graphene sheet adsorbed onto the substrate. Therefore, this paper indicates the possibility of the identification of the lattice orientation and the edge structure of the graphene sheet.

In this paper, we discussed the importance of the dynamics of the free edge during the peeling process. On the other hand, we also found the importance of the shape of the graphene sheet. Additional simulated model and results are shown in Figure 17. As shown in Figure 17(a), rectangular graphene sheet whose aspect ratio is different from that of the graphene sheet of Figure 1(b) is used. The basic shape of the vertical force curve, Figure 17(b), is similar to that of Figure 3. However, the armchair-type edge is peeled first for Figure 10(a), although the zigzag-type edge is peeled first for Figure 1(b) as discussed in Section III. This means that the shape of the graphene sheet plays an important role for deciding which edge is peeled first. Effect of the graphene shape on the peeling process will be discussed in detail somewhere in the near future.

Lastly it should be noted that the peeling process discussed in this paper is closely related to the atomic-scale wear of the graphite and the graphene tip formation in the friction force microscopy [22]. When the tip is pushed onto the surface for less than the critical tip height, the outermost graphene layer is attached to the FFM tip, which results in the formation of the graphene tip. In that case, the graphene sheet takes the surface contact with the second layer graphene, and it takes the two-dimensional stick-slip motion. However, it is difficult to observe directly the stickslip motion during the scan process, due to the very small gap between the FFM tip and the graphite surface. On the other hand, if the peeling process is used, it can be expected that the contact at the AFM tip/graphite interface has a wider space to be observed directly by ex Transmission Electron Microscopy (TEM). This paper indicates the possibility of a direct observation of the stick-slip motion of the graphene sheet, that is to say, the elementary process of the atomic-scale friction or superlubricity which occurs at the tip/graphite surface interface.

\section{Acknowledgments}

This research was supported by a Grant-in-Aid for Scientific Research (B) from the Japan Society for the Promotion of Science (no. 20360022). This work was also supported by the Ministry of Education, Culture, Sports, Science and Technology through a Grant-in-Aid for Building Strategic Research Infrastructures.

\section{References}

[1] N. Sasaki, A. Toyoda, H. Saitoh, N. Itamura, M. Ohyama, and K. Miura, "Theoretical simulation of atomic-scale peeling of single-walled carbon nanotube from graphite surface," $e$ Journal of Surface Science and Nanotechnology, vol. 4, pp. 133137, 2006.

[2] N. Sasaki, A. Toyoda, N. Itamura, and K. Miura, "Simulation of nanoscale peeling and adhesion of single-walled carbon nanotube on graphite surface," e-Journal of Surface Science and Nanotechnology, vol. 6, pp. 72-78, 2008.

[3] N. Sasaki, H. Saitoh, N. Itamura, and K. Miura, "Analysis of lateral orientation of single-walled carbon nanotube on graphite," e-Journal of Surface Science and Nanotechnology, vol. 7, pp. 48-52, 2009.

[4] M. Ishikawa, R. Harada, N. Sasaki, and K. Miura, "Visualization of nanoscale peeling of carbon nanotube on graphite," Applied Physics Letters, vol. 93, no. 8, Article ID 083122, 2008.

[5] M. Ishikawa, R. Harada, N. Sasaki, and K. Miura, "Adhesion and peeling forces of carbon nanotubes on a substrate," Physical Review B, vol. 80, no. 19, Article ID 193406, 2009.

[6] K. Autumn, Y. A. Liang, S. T. Hsieh et al., "Adhesive force of a single gecko foot-hair," Nature, vol. 405, no. 6787, pp. 681$685,2000$.

[7] K. Autumn, M. Sitti, Y. A. Liang et al., "Evidence for van der Waals adhesion in gecko setae," Proceedings of the National Academy of Sciences of the United States of America, vol. 99, no. 19, pp. 12252-12256, 2002.

[8] L. Qu, L. Dai, M. Stone, Z. Xia, and Z. L. Wang, "Carbon nanotube arrays with strong shear binding-on and easy normal lifting-off," Science, vol. 322, no. 5899, pp. 238-242, 2008.

[9] K. S. Novoselov, A. K. Geim, S. V. Morozov et al., "Electric field in atomically thin carbon films," Science, vol. 306, no. 5696, pp. 666-669, 2004.

[10] K. S. Novoselov, A. K. Geim, S. V. Morozov et al., "Twodimensional gas of massless Dirac fermions in graphene," Nature, vol. 438, no. 7065, pp. 197-200, 2005.

[11] A. K. Geim and K. S. Novoselov, "The rise of graphene," Nature Materials, vol. 6, no. 3, pp. 183-191, 2007.

[12] M. Ishikawa, N. Sasaki, and K. Miura, in preparation.

[13] N. Sasaki, H. Okamoto, N. Itamura, and K. Miura, "Peeling of graphene sheet-simulation study," e-Journal of Surface Science and Nanotechnology, vol. 7, pp. 783-786, 2009.

[14] N. Sasaki, H. Okamoto, N. Itamura, and K. Miura, "Atomicscale friction of monolayer graphenes with armchair- and zigzag-type edges during peeling process," e-Journal of Surface Science and Nanotechnology, vol. 8, pp. 105-111, 2010. 
[15] J. Tersoff, "Empirical interatomic potential for carbon, with applications to amorphous carbon," Physical Review Letters, vol. 61, no. 25, pp. 2879-2882, 1988.

[16] W. H. Press, S. A. Teukolsky, W. T. Vetterling, and B. P. Flannery, Numerical Recipes: The Art of Scientific Computing, Cambridge University Press, New York, NY, USA, 2nd edition, 1992.

[17] J. P. Lu, X.-P. Li, and R. M. Martin, "Ground state and phase transitions in solid $\mathrm{C}_{60}$," Physical Review Letters, vol. 68, no. 10, pp. 1551-1554, 1992.

[18] S. D. Stoddard and J. Ford, "Numerical experiments on the stochastic behavior of a lennard-jones gas system," Physical Review A, vol. 8, no. 3, pp. 1504-1512, 1973.

[19] N. Sasaki, N. Itamura, and K. Miura, "Simulation of atomicscale ultralow friction of graphite/ $\mathrm{C}_{60} /$ graphite interface along

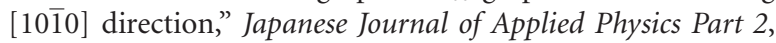
vol. 46, no. 45-49, pp. L1237-L1239, 2007.

[20] N. Itamura, K. Miura, and N. Sasaki, "Analysis of mechanism of low lateral stiffness of superlubric $\mathrm{C}_{60}$ bearing system," Japanese Journal of Applied Physics, vol. 48, no. 3, Article ID 030214, 2009.

[21] N. Itamura, K. Miura, and N. Sasaki, "Simulation of scandirectional dependence of superlubricity of $\mathrm{C}_{60}$ molecular bearings and graphite," Japanese Journal of Applied Physics, vol. 48, no. 6, Article ID 060207, 2009.

[22] N. Sasaki, H. Saitoh, K. Terada, N. Itamura, and K. Miura, "Simulation of atomic-scale wear of graphite-nanotip induced graphene formation," e-Journal of Surface Science and Nanotechnology, vol. 7, pp. 173-180, 2009. 

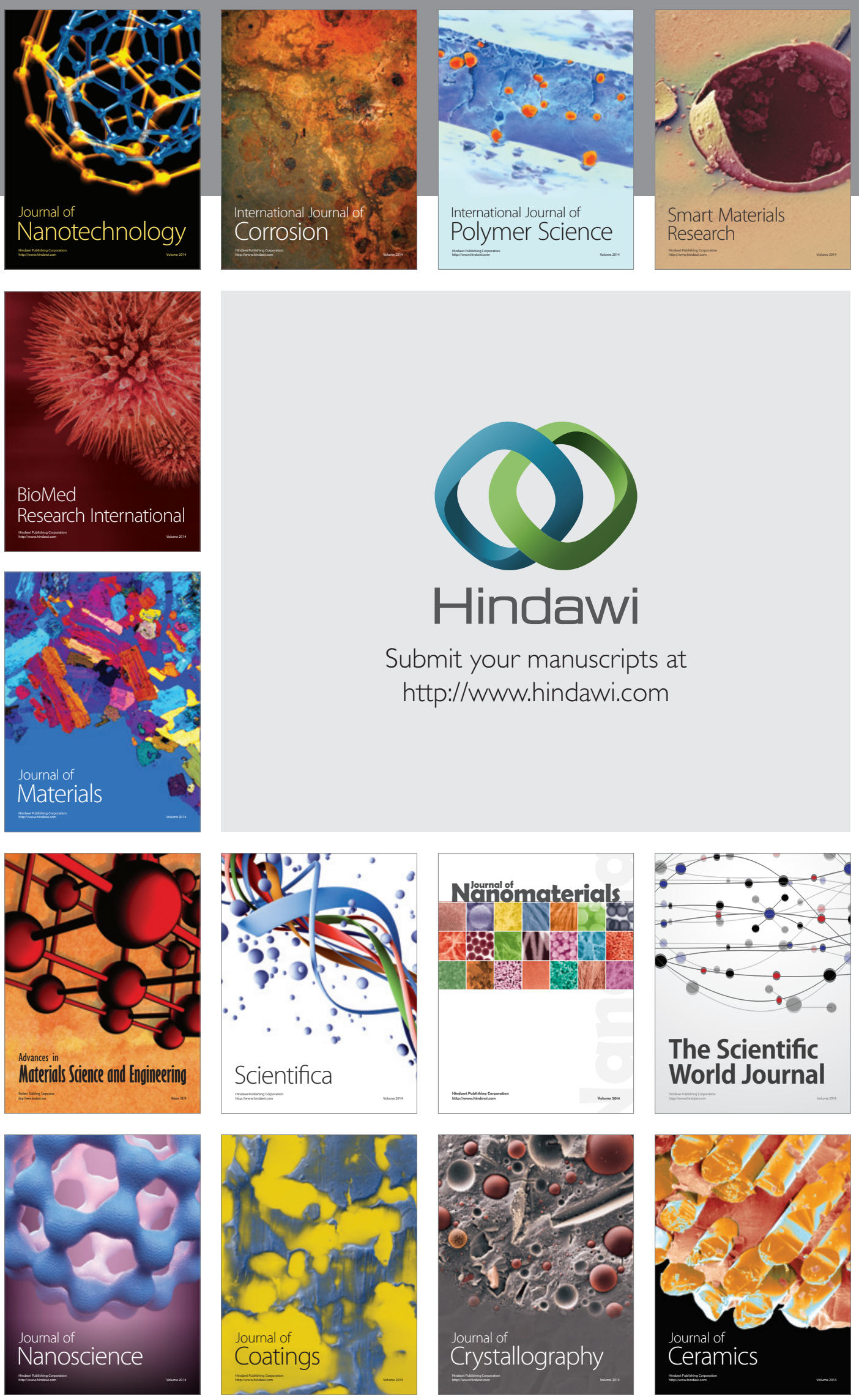

The Scientific World Journal

Submit your manuscripts at

http://www.hindawi.com

\section{World Journal}

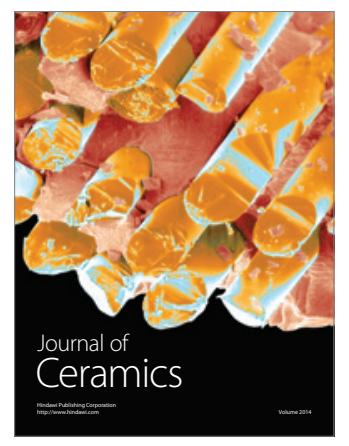

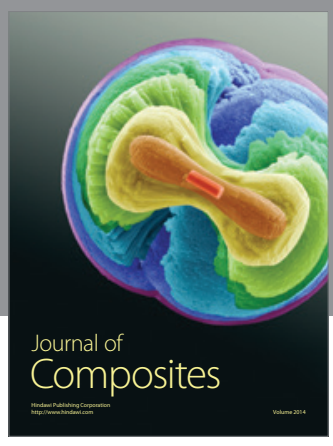
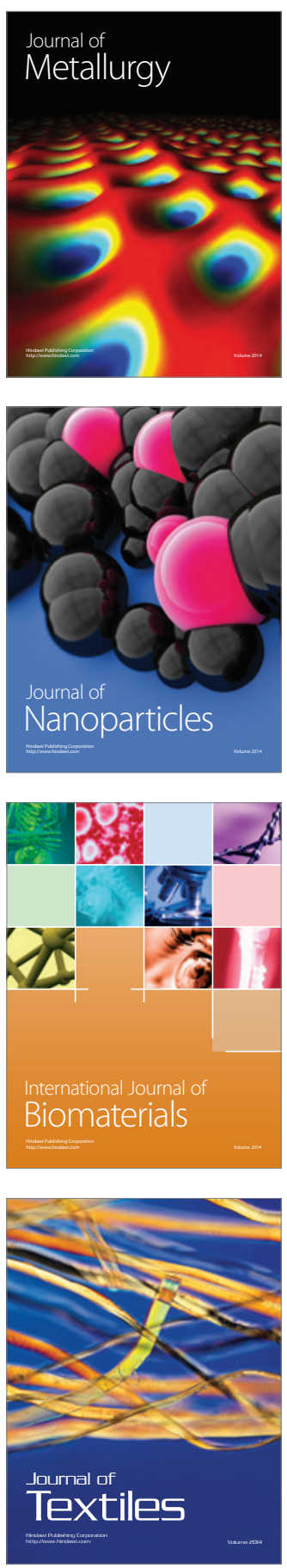